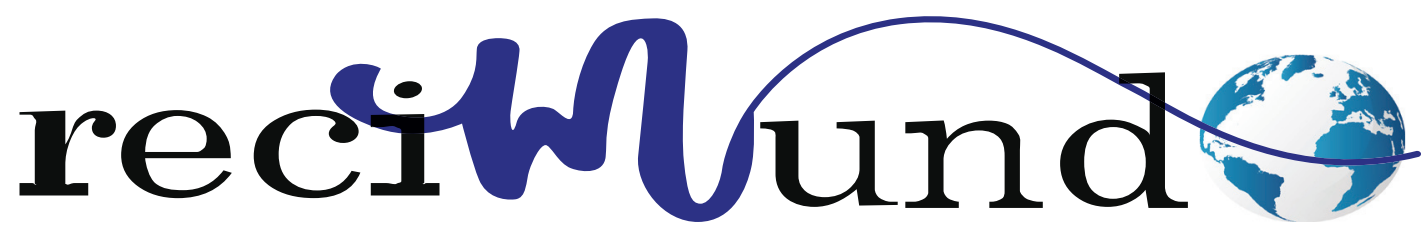

Revista Científica Mundo de la Investigación y el Conocimiento

DOl: 10.26820/recimundo/4.(4).noviembre.2020.19-29

URL: http://recimundo.com/index.php/es/article/view/923

EDITORIAL: Saberes del Conocimiento

REVISTA: RECIMUNDO

ISSN: 2588-073X

TIPO DE INVESTIGACIÓN: Artículo de revisión

CóDIGO UNESCO: 5801 Teoría y Métodos Educativos

PAGINAS: $19-29$

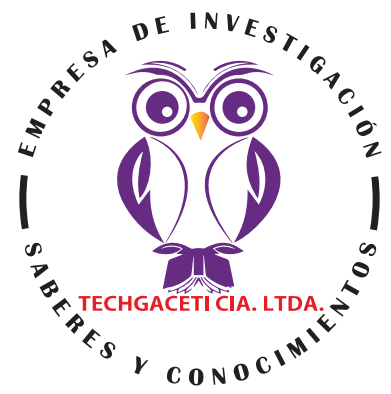

\title{
Las bases de datos como estrategia didáctica para investigadores noveles
}

Databases as a didactic strategy for novel researchers Bancos de dados como estratégia didática para novos pesquisadores

Peraza de Aparicio Cruz Xiomara'; Zurita Barrios Nhaylett Yoskyra²

RECIBIDO: 10/07/2020 ACEPTADO: 26/09/2020 PUBLICADO: 10/11/2020

1. PhD en Ciencias de la Educación; PhD en Desarrollo Social; MSc Educación Superior Mención Docencia; Espec Medicina Familiar; Médico Cirujano; Universitaria; Coordinadora de la Carrera Cultura Tradicional de la Salud en la Universidad Metropolitana; Actualmente docente de la carrera de Enfermería Coordinadora de los proyectos de Vinculación e Investigación de la carrera Enfermería; Quito, Ecuador; (iD https://orcid.org/0000-0003-2588-970X

2. Doctorante en Ciencias de la Educación de la Universidad Fermín Toro; Espec en Cuidados Críticos Adulto; Espec en Cuidados Críticos; Neonatales; MSc Gerencia en Salud Pública; Licda en Enfermeria; Docente de la carrera Enfermeria Universidad Metropolitana; Quito, Ecuador; (iD https://orcid.org/0000-0002-1542-3351

CORRESPONDENCIA

Peraza de Aparicio Cruz Xiomara

cperaza@umet.edu.ec

Guayaquil, Ecuador

¡ RECIMUNDO; Editorial Saberes del Conocimiento, 2020 


\section{RESUMEN}

Las TIC ofrecen para la investigación las bases de datos académicas que aportan información relacionada con revistas, libros, ponencias e informes de investigación, artículos científicos, tesis y cualquier otro tipo de investigaciones, que pueden ser consultadas desde las bibliotecas digitales de las universidades El Objeto de estudio: Conocer las bases de datos y la forma de acceder a esta, las cuales conforman estrategias didácticas que se convierten en oportunidades y opciones diversas para fortalecer su capacidad investigativa. Materiales y Métodos: se realizó una investigación documental, se hizo consulta de artículos científicos en bases indexadas desde 2016 en adelante. Se revisaron textos y documentos sobre el uso de las Tics en la formación de investigadores noveles Conclusiones: De todo el material consultado se concluye en los aportes de Siemens desde el conectivismo para sustentar el uso de las redes para describir el conocimiento y el aprendizaje, su influencia en el manejo de las bases de datos como Estrategia Didactica para la formación de los investigadores noveles en las carreras de salud.

Palabras clave: Bases de datos, Investigación, Conectivismo, Didáctica, Redes.

\section{ABSTRACT}

TICs offer academic databases for research that provide information related to journals, books, papers and research reports, scientific articles, theses and any other type of research, which can be consulted from the digital libraries of the El Objeto universities Study: Know the databases and how to access them, which make up didactic strategies that become opportunities and diverse options to strengthen their research capacity. Materials and Methods: a documentary research was carried out; scientific articles were consulted in indexed databases from 2016 onwards. Texts and documents on the use of ICTs in the training of new researchers were reviewed Conclusions: From all the material consulted, it is concluded in the contributions of Siemens from connectivism to support the use of networks to describe knowledge and learning, its influence on the management of databases as a Didactic Strategy for the training of new researchers in health careers

Keywords: Databases, Research, Connectivism, Didactics, Networks.

\section{RESUMO}

Os TICs oferecem bancos de dados acadêmicos para pesquisas que fornecem informações relacionadas a periódicos, livros, artigos e relatórios de pesquisa, artigos científicos, teses e qualquer outro tipo de pesquisa, que podem ser consultados nas bibliotecas digitais das universidades El Objeto. Estudo: Conheça os bancos de dados e como acessá-los, que constituem estratégias didáticas que se transformam em oportunidades e opções diversas para fortalecer sua capacidade de pesquisa. Materiais e Métodos: foi realizada uma pesquisa documental; os artigos científicos foram consultados em bases de dados indexadas a partir de 2016. Foram revisados textos e documentos sobre o uso das TICs na formação de novos pesquisadores. Conclusões: De todo o material consultado, conclui-se nas contribuições da Siemens do conectivismo para apoiar o uso de redes para descrever o conhecimento e a aprendizagem, sua influência na gestão de bases de dados como Estratégia Didática para a formação de novos pesquisadores na área da saúde.

Palavras-chave: Bancos de dados, Pesquisa, Conectivismo, Didática, Redes. 


\section{Introducción}

Para progresar con éxito en un mundo que evoluciona constantemente y resolver los problemas cotidianos de manera eficaz, los individuos, las comunidades y los países deben dotarse de ciertas competencias que les permitan buscar información, evaluarla de manera crítica y transformarla en conocimientos nuevos. Cuando se dominan las competencias básicas en materia de información, se tiene acceso a nuevas oportunidades que aumentan la calidad de vida. La alfabetización informacional está estrechamente ligada a otras competencias como son los conocimientos básicos en Tecnología de la Información y de la Comunicación, y la alfabetización digital y mediática, estas pueden complementar, enriquecer y transformar la educación.

En su calidad de Organización principal de las Naciones Unidas para la Educación, la UNESCO (1) orienta el quehacer internacional con miras a ayudar a los países a entender la función que puede desarrollar esta tecnología en acelerar el avance hacia el Objetivo de Desarrollo Sostenible (ODS4), una visión plasmada en la Declaración de Qingdao (2).

La UNESCO comparte los conocimientos respecto a las diversas formas en que la tecnología puede facilitar el acceso universal a la educación, reducir las diferencias en el aprendizaje, apoyar el desarrollo de los docentes, mejorar la calidad y la pertinencia del aprendizaje, reforzar la integración y perfeccionar la gestión y administración de la educación.

La Organización examina el mundo en busca de ejemplos exitosos de aplicación de las TIC a la labor pedagógica - ya sea en escuelas primarias de bajos recursos, universidades en países de altos ingresos o centros de formación profesional - con miras a elaborar políticas y directrices.
Mediante actividades de fomento de la capacidad, asesoramiento técnico, publicaciones, investigaciones y conferencias internacionales como la Conferencia internacional sobre la Inteligencia Artificial en la Educación o la Semana del Aprendizaje Mediante Dispositivos Móviles, (3) la UNESCO ayuda a los gobiernos y a otras partes interesadas a valerse de las tecnologías para fomentar el aprendizaje.

Hoy por hoy se exige una alfabetización informacional que significa que, no solo se debe saber que la información existe, sino conocer cómo buscar, encontrar, utilizar y manipular una información para que se convierta en conocimiento individual y social. La tecnología aplicada al cuidado humano ofrece métodos y técnicas que fortalecen la práctica, por lo que su uso es necesario, pero por muy elemental que sea, se debe saber utilizar y comprender sus aplicaciones para que pase a formar parte del proceso de cuidado y haga la labor de Enfermería más eficiente.

Es necesario que los profesionales de enfermería reconozcan la necesidad de desarrollar habilidades relacionadas con el manejo de información para desempeñar con éxito su trabajo en este novedoso escenario de actuación como investigadores. Además, la práctica de la enfermería basada en la evidencia (EBE) conduce a tomar decisiones clínicas a partir de la información obtenida de publicaciones científicas, por lo que, no solo hay que saber reconocer la evidencia científica en las publicaciones, sino acceder también a ellas.

Los profesionales de enfermería dedican la mayor parte de su ejercicio a la labor asistencial y de gestión del cuidado, sin dar importancia a la necesidad de meditar sobre las bases y cimientos deontológicos de la profesión de enfermería, y considerar las responsabilidades éticas y jurídicas, todo lo cual obliga a ejercer una labor profesional consecuente a éstas. Esto implica una per-

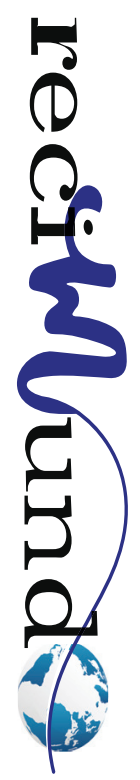


manente autoevaluación y mejoramiento de su labor asistencial y a la vez, fundamentar los procedimientos en la evidencia científica, para lo cual se requiere el ejercicio del espíritu investigativo, apoyado por la actualización permanente en el estudiante en el campo de la investigación, por ello el estudio tiene como objetivo :Conocer las bases de datos y la forma de acceder a esta, las cuales conforman estrategias didácticas que se convierten en oportunidades y opciones diversas para fortalecer su capacidad investigativa.

\section{Las bases de datos}

Una base de datos (Database) almacena datos y los conecta en una unidad lógica junto a los metadatos necesarios para su procesamiento. Estas según Sánchez (4), son instrumentos de gran utilidad para gestionar grandes ficheros y facilitar la consulta de información. En muchas, además, puede definirse un esquema de permisos que establece qué personas o programas pueden acceder a los datos, y a cuáles, con el objetivo de presentar el contenido de forma adecuada y clara.

Los distintos sistemas de bases de datos se diferencian conceptualmente entre sí y tienen, por lo tanto, sus propias ventajas y desventajas. Pero, antes que nada, es conveniente diferenciar entre la base de datos en sí y el sistema que la gestiona. Como base de datos se designa al conjunto de los datos que se ha de ordenar, mientras que el sistema de gestión de la base de datos (SGBD) es responsable de su administración, determinando así su estructura, el orden, los permisos de acceso, las dependencias, entre otros. (4) Para ello acostumbra a utilizar un compilador propio y un modelo adecuado de base de datos que determina la arquitectura del sistema de base de datos.

En muchos casos, solo ciertas aplicaciones, o aquellas que han sido exactamente definidas para ello, pueden leer estos sistemas. Es aquí donde, con frecuencia, se dan confusiones terminológicas cuando un programa de base de datos se define solo como "base de datos". El término, además, se utiliza para referirse a simples colecciones de archivos, mientras que, en su sentido estricto, una carpeta con archivos en un ordenador no constituye una base de datos.

El modelo que goza de más popularidad a día de hoy es el relacional, aunque tampoco queda libre de crítica. Su correspondiente sistema de gestión es más conocido como SGBDR (RDBMS en inglés) y como lenguaje utiliza normalmente SQL. (5) Este modelo basado en tablas, gira en torno al concepto de relación, un término bien definido en matemáticas y que aquí se utiliza como sinónimo de tabla. Para formular las relaciones se utiliza álgebra relacional, con cuya ayuda puede obtenerse la información de estas relaciones. Este es el principio que fundamenta el lenguaje SQL.

La administración de un Sistema de Base de Datos (5), es un elemento importante en un sistema complejo como es la organización del motor de Base de Datos. Como elemento importante también es el modelado de datos donde se define como se tienen que organizar los datos y que SGDB se ajusta a las necesidades de la organización.

Es importante que los profesionales en formación de Enfermería conozcan las nociones básicas sobre el proceso que desarrollan los especialistas en información, estos se han dado a la tarea de hacer estudios para entregar como productos a sus usuarios, informes de inteligencia que los ayuden a comprender los aspectos más relevantes para su realidad y que les sean factibles para la toma de decisiones.

La profesión de analista emerge entonces con especial énfasis en un tiempo cuyo signo es la abundancia de datos e informaciones que complican los procesos decisionales y donde los escépticos no se atreven 
a entender la esencia de su carácter. Se analizan las fuentes, en base a las necesidades de los usuarios, que deben estar en consonancia con los objetivos del consultante. Además, en un análisis de este tipo se deben validar las fuentes a utilizar, pues resulta muy importante que la información a presentar sea confiable y actualizada; para que los resultados obtenidos puedan ser utilizados adecuadamente en la toma de decisiones pero que además ofrezca a los usuarios alternativas de decisión.

Los objetivos fundamentales de los SBD (6) comprenden los siguientes:

1. Independencia de los datos y los programas de aplicación. Para mayor eficiencia esta independencia de los mismos les da inmunidad a las aplicaciones frente a los cambios en la estructura de almacenamiento y en la estrategia de acceso, constituyendo el objetivo fundamental de los SBD.

2. Minimización de la redundancia. Trata de lograr la eliminación de la redundancia superflua.

3. Integración y sincronización de las bases de datos. La integración consiste en garantizar una respuesta a los requerimientos de diferentes aspectos de los mismos datos por diferentes usuarios, de forma que, aunque el sistema almacene cierta estructura y cierto tipo de representación, debe garantizar entregar al programa de aplicación datos que solicita y en la forma en que se lo solicita.

4. Integridad de los datos. Debe garantizar la no contradicción entre los datos almacenados, de modo que, en cualquier momento del tiempo, los datos sean correctos.

5. Seguridad y protección de los datos. Protección: garantizar el acceso autorizado a los datos. Seguridad: que el sistema de base de datos disponga de métodos que garanticen la restauración de las Bases de Datos al producirse alguna falla técnica o interrupciones eléctricas.

6. Control centralizado. Permite controlar de manera sistemática y única laos datos que se almacenan en la Base de Datos.

Un sistema de base de datos se encuentra dividido en módulos cada uno de los cuales controla una parte de la responsabilidad total de sistema. En la mayoría de los casos, el sistema operativo proporciona únicamente los servicios más básicos y el sistema de la base de datos debe partir de esa base y controlar además el manejo correcto de los datos. Así el diseño de un sistema de base de datos debe incluir la interfaz entre el sistema de base de datos y el sistema operativo.

Los componentes funcionales (6) de un sistema de base de datos, son:

\section{Gestor de archivos}

Gestiona la asignación de espacio en la memoria del disco y de las estructuras de datos usadas para representar información.

Manejador de base de datos.

Sirve de interfaz entre los datos y los programas de aplicación.

Procesador de consultas.

Traduce las proposiciones en lenguajes de consulta a instrucciones de bajo nivel. Además, convierte la solicitud del usuario en una forma más eficiente.

Compilador de DDL.

Convierte las proposiciones DDL en un conjunto de tablas que contienen metadatos, estas se almacenan en el diccionario de datos. 
Archivo de datos.

En él se encuentran almacenados físicamente los datos de una organización.

Diccionario de datos.

Contiene la información referente a la estructura de la base de datos.

Índices.

Permiten un rápido acceso a registros que contienen valores específicos.

\section{Competencias en la formación para la in- vestigación}

Las competencias pueden definirse de diferentes maneras, para unos es la característica subyacente en un individuo que esta causalmente relacionada a un estándar de efectividad y / o a una performance superior en un trabajo o situación (7) (8). Para otros autores las competencias son aquellas habilidades, capacidades y conocimientos que una persona tiene para cumplir eficientemente determinada tarea, se direccionan a un determinado campo definen el pensamiento, el carácter, los valores y el buen manejo de las situaciones problemáticas. Las mismas son adquiridas mediante el aprendizaje y la formación de las personas; siendo vitales para el ejercicio en el campo donde son necesarias dichas competencias.

La competencia investigativa va de la mano con la crítica y la reflexión. (9) La crítica en los jóvenes investigadores está manifestada en su capacidad de pensar, analizar, tener sensibilidad de su entorno social, sospechar de las soluciones para volver a observar el desarrollo y cómo se llegó a esas conclusiones, es decir, ser capaz de cuestionar el mundo que le rodea. De este modo, los jóvenes ordenan su pensamiento y argumentan con los demás sus pareceres en relación con sí mismos, con los otros y con lo otro.

La competencia investigativa está asociada directamente al desempeño, expresado concretamente en la manifestación de los recursos con que cuenta el profesional de la Enfermería para realizar una tarea o actividad, enfrentar y resolver una situación de manera

particular y crítica; por lo que no puede hablarse de esta competencia como virtualidad, sino como acción, ya que la relación entre conocimiento y actuación debe ser biunívoca y evidenciada en contextos específicos.

Los procesos de formación de la competencia investigativa de los profesionales de la Enfermería emergen para hacer de la atención de salud (10) un servicio más pertinente a las demandas sociales, donde adquieren un significado el saber y el qué, el saber hacer y el cómo y el saber ser y saber ser para quién, capaz de operar con eficacia en el contexto específico de las dificultades y los retos propios que enfrenta el país.

La calidad de la docencia del profesional de enfermería debe garantizar la preparación de los futuros profesionales competentes que se identifiquen con su quehacer y que estén alertas a reaccionar al medio y las exigencias de los servicios de salud. (11) Enfermería actualmente ha encontrado en sus quehaceres, oportunidades que la han posicionado como una profesión con bases científicas sólidas, siendo la investigación una herramienta indispensable para el progreso.

En la Enfermería actual, a nivel internacional, existe una gran necesidad de adoptar una práctica basada en la evidencia, implementarla, enseñarla, estudiarla y estandarizarla. La práctica basada en la evidencia, es la utilización de la mejor evidencia disponible para mejorar los resultados e informar la práctica clínica, implica diferentes elementos, incluyendo una orientación hacia una autoevaluación crítica, la producción de evidencia a través de la investigación, su aplicación en la práctica diaria de la enfermería, por estas razones en 
enfermería, es muy importante incrementar la producción científica, que permita su consolidación como ciencia (12), en este sentido, los grupos de investigación son estrategias importantes para fomentar el pensamiento crítico, reflexivo e investigativo de los estudiantes desde su formación.

La investigación en enfermería es la fuente de desarrollo del conocimiento, que le da la característica de la disciplina, que ha de estar presente tanto en la práctica clínica como comunitaria y en el desarrollo de la administración y la educación. La sociedad de Honor de Enfermería, Sigma Theta Tau International Honor Society of Nursing (13), fue la primera organización en Estados Unidos que dio fondos para la investigación en enfermería en 1936. Hoy en día, esta organización internacional proporciona financiación para la investigación de los profesionales, a través de la recaudación de fondos y la gestión activa. (11)

\section{Las bases de datos y su importancia en la investigación}

Las bases de datos de investigación ofrecen a los usuarios acceso a miles de libros, artículos de revistas, imágenes, cuadros y fuentes primarias. Estas bases de datos contienen artículos académicos y revisados por autores escritos por autores creíbles, como periodistas, investigadores y expertos en su campo. Dado que las bases de datos proporcionan herramientas de búsqueda eficaces para reducir los resultados, los usuarios pueden encontrar más rápidamente la información que necesitan.

Cabe señalar que, aunado a la puesta en práctica de los microprocesadores, a mediados de la década de los ochenta la elaboración de bases de datos facilitadas por los especialistas en computación hicieron posible la masificación del uso de las bases de datos, este complejo proceso deja de ser un trabajo inalcanzable para una gran cantidad de centros de investigación, ya que conocían las ventajas de acumular y cruzar la información sobre un tema con otro tema, de un país en especial, de un periodo determinado. Además, en ese mismo tiempo en las bibliotecas se visualizan las posibilidades que les brinda la computación y generan infinidad de bases de datos referenciales, que guardan un alto grado de homogeneidad debido a las normas y criterios internacionales creados con anterioridad para la clasificación de todo tipo de documentos.

Un Sistema Gestor de Bases de Datos (SGBD) o DBMA (DataBase Management System) consiste en una colección de programas cuyo objetivo es servir de interfaz entre la base de datos, el usuario y las apps. (14). También se dice que es un conjunto de aplicaciones informáticas que permite manejar las bases de datos. Este tipo de programas sirven para que los usuarios y las bases de datos se puedan comunicar de forma sencilla, no solo comprenden los programas, sino que también incluyen los propios datos almacenados que normalmente se encuentran relacionados.

Está compuesto por:

- Lenguaje de definición de datos

- Lenguaje de manipulación de datos

- Lenguaje de consulta.

Actualmente, conviven multitud de SGBD en el mercado y suelen ser clasificados en base a la forma en que administran los datos en:

- Relacionales (SQL)

- No relacionales (NoSQL)

\section{Sistemas Gestores de Bases de Datos Relacionales (SQL)}

Este modelo establece relaciones o vínculos entre datos. Cada relación representa un conjunto de entidades con las mismas propiedades y se compone de una serie de filas o registros (las llamadas tuplas), cuyos

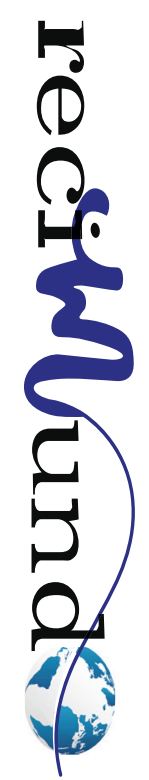


valores dependen de ciertos atributos (columnas).

Los principales SGBD SQL son: MySQL, MariaDB, SQLite (biblioteca escrita en C que implementa un SGBD), PostgreSQL, Microsoft SQL Server y Oracle.

\section{Sistemas Gestores de Bases de Datos No Relacionales (NoSQL)}

Una base de datos no relacional no requiere de estructuras de datos fijas (tablas), no da completas garantías de las características ACID y realiza escala horizontal de forma óptima.

Se hace uso de ellas en entornos distribuidos que deban estar continuamente disponibles y operativos y que además gestionan un gran volumen de datos.

Los SGBD NoSQL más utilizados actualmente para administrar este tipo de bases de datos son: MongoDB, Redis y Cassandra.

Para efectos de este trabajo se consideran las bases de datos relacionales (14), el modelo relacional para el modelado y la gestión de bases de datos, es un modelo de bases de datos basado en la lógica de predicados y en la teoría de conjuntos. Estos sistemas solucionan los problemas de redundancia y no integración de los datos que tenían otros sistemas más antiguos. La idea fundamental de este modelo son las relaciones. Esta organización relacional se caracteriza por unas tablas de bases de datos con estructura de matriz, donde las filas son los registros y las columnas son los campos.

Este modelo tiene entre sus ventajas se encuentran:

- Poseen herramientas que garantizan la no duplicidad de registros

- Garantiza la integridad referencial, al eliminar un registro elimina todos los registros dependientes del mismo
- Favorece la normalización de la BBDD

- Mejora la potencia de las consultas, al poder utilizar varias tablas.

Entre los inconvenientes del modelo se encuentran:

- Dificultad para manejar los bloques de texto y los tipos de datos

- Presentan deficiencias con archivos multimedia y gráficos.

Actualmente existen multitud de bases de datos. En el caso de las ciencias de la salud las tres bases de datos más importantes a nivel mundial serien: Pubmed, Cinahl y Scopus.

En primer lugar, es importante diferenciar entre Pubmed y Medline.

Medline es la base de datos más popularmente utilizada a nivel mundial en ciencias de la salud. Esta producida por la librería nacional de medicina de los Estados Unidos. (US National Library of Medicine) y data de los años 60. Abarca las disciplines de medicina, enfermería, veterinaria, administración sanitaria y ciencias preclínicas.

Pubmed es un sistema de búsqueda libre y gratuito a la base de datos Medline. Además de ofrecer acceso a todo el contenido de Medline da acceso a citas in process, artículos ahead of print, libros disponibles en el ncbi bookshelf, revistas adicionales pertenecientes a ciencias de la vida y artículos anteriores a 1966.

Pubmed está disponible desde el año 1996 y ha sido desarrollada por el National Center for Biothecnology Informational (NCBI). Actualmente Pubmed tiene aproximadamente más de 25 millones de referencias en permanente aumento, de hecho, se actualiza de forma semanal. PubMed® comprende más de 30 millones de citas de literatura biomédica de MEDLINE, revistas de ciencias biológicas y libros en línea. Las citas 
pueden incluir enlaces a contenido de texto completo de PubMed Central y sitios web de editores. (15)

En cuanto a Cinahl, es la base de datos más importante de enfermería. Se denomina con las siglas en inglés: Cumulative Index to Nursing and Allied Health Literature (Índice Acumulativo de Enfermería y Literatura de Ciencias de la Salud) (16), junto con PubMed es una base datos imprescindible en ciencias de la salud, ya que maneja una gran cantidad de información. Cinhal está producida por EBSCO publishing, y es una base de datos de subscripción. Por lo tanto, el acceso no es gratuito y estará supeditado a que nuestra institución este subscrita.

En Cinhal Plus el contenido está dirigido a biomedicina, medicina alternativa y especialmente enfermería, desde 1982 hasta la actualidad. Una parte del contenido de Cinahl plus se solapa con el de Pubmed, pero eso no significa que se pueda substituir una base de datos por otra, ya que en cada una hay contenida información específica.

CINAHL Plus proporciona texto completo de más de 770 revistas indexadas en CINA$\mathrm{HL}$ y también proporciona el texto completo de más de 275 libros / monografías.

Por lo tanto, por la gran cantidad de información que contiene, CINAHL Plus es una base de datos de uso prácticamente obligatorio para las búsquedas bibliográficas en ciencias de la salud, especialmente en enfermería.

Por último, pero no menos importante, Scopus. (17) Scopus es la base de datos más nueva de las tres y posiblemente también la más potente en cuanto a contenido.

Scopus está producida por el editorial Elsevier y el acceso al igual que Cinhal también es mediante subscripción.

Es importante tener en cuenta que Scopus contiene el $100 \%$ del contenido de Medline, pero es que además es la mayor base de resúmenes y referencias bibliográficas de literatura científica revisada por pares, conteniendo más de 18.000 títulos de 5.000 editoriales Internacionales.

También contiene datos sobre patentes, fuentes de Internet de contenido científico, revistas científicas de acceso abierto y memorias de congresos y conferencias.

Mencionar también que contiene artículos in press de más de 3000 revistas y todos los contenidos se actualizan diariamente.

Por último y muy importante, Scopus es la única base de datos que dispone de herramientas de evaluación de la producción científica, como serian: perfil de autor, perfil de institución, rastreador de citas, índice h y analizador de revistas científicas.

\section{Conclusiones}

-La alfabetización informacional significa que, no solo se debe saber que la información existe, sino conocer cómo buscar, encontrar, utilizar y manipular una información para que se convierta en conocimiento individual y social para ser puesta al servicio de la investigación en las áreas de la salud.

-Los distintos sistemas de bases de datos se diferencian conceptualmente entre sí y tienen, por lo tanto, sus propias ventajas y desventajas. Pero, antes que nada, es conveniente diferenciar entre la base de datos en sí y el sistema que la gestiona. Actualmente existen multitud de bases de datos. En el caso de las ciencias de la salud las tres bases de datos más importantes a nivel mundial serien: Pubmed, Cinahl y Scopus.

- La revisión de bases de datos es una Estrategia Didactica practica en la formación de investigadores noveles es importante para que los docentes puedan generar nuevo conocimiento a partir de investigaciones realizadas en el contexto educativo para

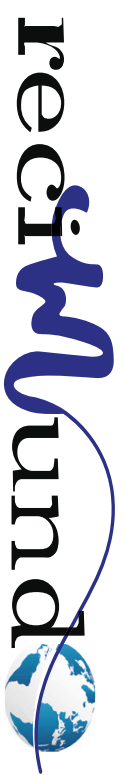


dar cuenta del impacto de sus propuestas y estrategias metodológicas y así, de esta manera, otros docentes y profesionales que acompañen procesos de formación lograrían nutrir su banco de propuestas metodológicas y sus prácticas significativas, a fin de lograr un impacto en la calidad educativa, en este caso sobre las áreas de salud desarrolladas por los profesionales de Enfermería, contribuyendo a mejorar el nivel de vida de la comunidad.

\section{Bibliografía}

Organización de las Naciones Unidas para la educación(UNESCO,2019). Las TIC en la educación. Disponible en: https://es.unesco.org/themes/ tic-educacion

Organización de las Naciones Unidas para la educación(UNESCO,2019). Apertura del Foro Mundial sobre TIC y Educación 2030 en Qingdao. Disponible en: http://www.unesco.org/new/es/media-services/single-view-tv-release/news/international_forum_on_ict_and_education_2030_opens_ in_qingd/

Organización de las Naciones Unidas para la educación(UNESCO,2019). Conferencia internacional sobre la Inteligencia Artificial en la Educación: Planificar la educación en la era de la IA: un paso más hacia adelante. Disponible en: https://es.unesco. org/themes/tic-educacion/conferencia-ia-educacion-2019

Sánchez, O. Aplicaciones informáticas de bases de datos relacionales. Microsoft Access 2016. Ediciones Paraninfo, S.A., 2016

Talledo, J. Administración y monitorización de los SGBD. Ediciones Paraninfo, S.A., 2016

Pisco A, Regalado J, Gutiérrez J, Quimis O, Marcillo K \& Marcillo J. Fundamentos sobre la Gestión de Base de Datos. Volumen23 de Ingeniería y Tecnología. 3Ciencias, 2017
McClelland. Testing for Competence Rather Than for "intelligence". Institute for Competence Assessment, McBer and Company 1971

Spencer, L \& Spencer, G. Competence at work. Models for superior performance. Nueva York: Wiley \& Sons

D'olivares, N \& Casteblanco, C. Competencias investigativas: inicio de formación de jóvenes investigadores en educación media. RHS. Revista. Humanismo. Soc. 7(1): 6-21, junio 2019

Mendoza, N; Chibas, Y; Lara, N; Basulto, V \& Albelo, M. Desarrollo de Competencias en los profesionales de Enfermería. Rev Inf Cient. Vol. 96, No. 3 (2017)

De Arco, O \& Suarez, Z. Rol de los profesionales de enfermería en el sistema de salud colombiano Univ. Salud. 20(2):171-182, 2018 (May - Ago)

Benítez J. La importancia de la investigación en Enfermería, Enferm Inv. 2019; 5(1):1-2

Sigma. Sigma Organizational Fact Sheet. Indianapolis: Sigma; 2017. [cited 2017 Abril 15]. Available from: https://www.nursingsociety.org/connect-engage/about-stti/sigma-theta-tau-international-organizational-fact-sheet

De La Peña O'Shea, S. SGDB e instalación. Ed Paraninfo

National Institutes of Health (NIH). Pubmed. Disponible en: https://pubmed.ncbi.nlm.nih.gov/

Cumulative Index to Nursing and Allied Health Literature (Cinhal). CINHAL a texto completo. Disponible en: https://canalbiblos.blogspot.com/2018/10/ cinhal-texto-completo.html

Fundación Española para la Ciencia y la Tecnología (FECYT) Scopus. Funcionalidades Avanzadas En Scopus.2018. Disponible en: https://www.recursoscientificos.fecyt.es/sites/default/files/scopus_ avanzado_mayo_18.pdf 


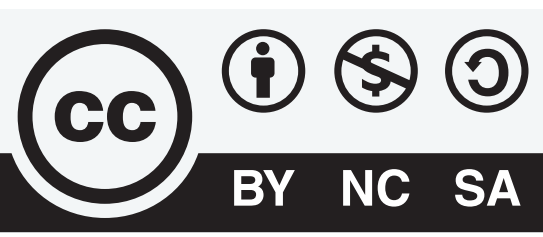

CREATIVE COMMONS RECONOCIMIENTO-NOCOMERCIAL-COMPARTIRIGUAL 4.0.

\section{CITAR ESTE ARTICULO:}

Peraza de Aparicio, C. X., \& Zurita Barrios, N. Y. (2020). Las bases de datos como estrategia didáctica para investigadores noveles. RECIMUNDO, 4(4). https://doi.org/10.26820/recimundo/4.

(4).noviembre.2020.19-29 